\title{
Ceftriaxone Induced Steven Johnson Syndrome: A Case Report
}

\author{
Asha K Rajan, Vedha pal Jeymani.S, Franklin Jose J, Deepak Paul Denagaran, Merlin Joan of Arc. M.C \\ Department of Pharmacy practice, Jaya College of Pharmacy, C.T.H. road, Thiruninravur, Chennai-602024, India.
}

*Corresponding author's E-mail: ashapharmd523@gmail.com

Received: 18-09-2020; Revised: 24-11-2020; Accepted: 30-11-2020; Published on: 15-12-2020.

\begin{abstract}
Steven Johnson Syndrome (SJS) is an idiosyncratic reaction characterized by severe hypersensitive reactions towards medications, presenting with exfoliative dermatitis, epidermal erythema and blistering. It may or may not be occasionally associated with Toxic Epidermal Necrolysis. Its clinical presentation includes mucocutaneous lesions and fever with necrolysis and sloughing of epidermis. Toxic Epidermal Necrolysis (TEN) which is similar to SJS presents with severe lesions widespread all over the body. The etiological causes of the above syndrome include infectious agents, drug-induced conditions, malignancy-related and idiopathic reactions. However, it resolves within few days to weeks or may extend to months or years. Drug-induced Steven Johnson Syndrome is more common and accounts for major patients, who may experience skin rashes, fever, red and purplish spots, itchy skin, eye redness, cough and mouth ulcer. The therapy mainly involves withdrawal of the culprit drugs and other non-essential medicines, IV fluids and bed care with wound dressing. Some of the drugs which can induce SJS are Carbamazepine, Ceftriaxone, Lamotrigine, Amikacin, Lumefantrine, Carboquone etc. The current study involves a specific drug Ceftriaxone which is a 3rd generation Cephalosporin broadspectrum antibiotic, commonly prescribed almost in all bacterial infections and prophylactic conditions. As per the pharmacist oath, a pharmacist ought to play a vital role in reducing the morbidity and mortality rate of SJS. The drug regimens should be strictly and continuously monitored to prevent further virulence.
\end{abstract}

Keywords: Ceftriaxone, Hypersensitivity, Stevens Johnson Syndrome, Toxic Epidermal Necrolysis.

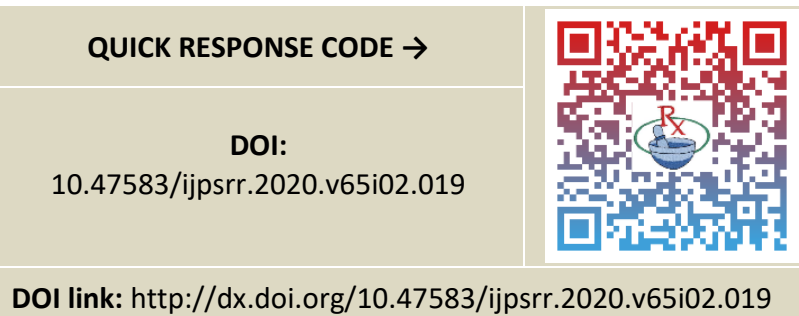

\section{INTRODUCTION}

D ay-to-day drug therapy includes many drugs prescribed for a single patient leading to polypharmacy and adverse drug reactions among the prescribed drugs and persisting disease conditions. SJS and TEN are two major life-threatening syndrome characterized by destruction of epidermis and mucosal layers of the skin, which may also be systemic and become generalized all over the body ${ }^{1-3}$. This syndrome is classified under the category of Drug-Induced Hypersensitivity Syndrome (DIHS). This type of cutaneous adverse drug reaction may produce immunosuppression which tends the patient to be more prone to other infectious diseases. SJS and TEN are life threatening conditions causing cell death with separation of epidermis from the dermis ${ }^{4-6}$. It mainly affects the skin and mucous membrane. The drugs inducing SJS are commonly and widely used, particularly the sulfa drugs. Although, this rare disease only have statistic of affecting 2 persons per million of general populations per year, it has a greater impact towards high mortality and morbidity ${ }^{7-9}$.
SJS presents in three different forms namely erythema multiforme, main form, toxic epidermal necrolysis (severe form). Clinical signs and presenting symptoms of SJS include mucous membrane erosion, positive Nikolsky sign, epidermal detachment, conjuctivitis, intense erythema, extreme pain, hemorrhagic crusting of lips and others like rash, fever, arthralgia, myalgia, rhinitis, headache, anorexia etc. triggering drugs for this conditions includes corticosteroids, NSAIDS, allopurinol, anticonvusants (valproic acid, Phenobarbital, carbamazepine), antibiotics (Aminopenicillins, quinolones, Cephalosporins, Tetracylines, imidazoles, antifungals) etc ${ }^{10-12}$.

The present study elaborates on a Ceftriaxone evoked Steven Johnson condition, which is mostly prescribed for bacterial infections of skin and mucous membranes, respiratory infections, urinary tract infections, sexually transmitted infections, etc. An individual should be made to administer the drug with caution and past drug consumption associated with allergies should be borne in mind to prevent the attack of SJS ${ }^{13-15}$. Test dose for hypersensitivity conditions could be carried on for examining on any allergic condition existing or not. Patients have to be informed that if any manifestation appears, quickly consult the physician or any health care professional near them, to withdraw the drug and not be continued thereafter ${ }^{2,3}$. 
Pathophysiology 5,7

Etiological causes mainly drugs

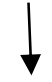

Failure to detoxify reactive

intermediate drug metabolite

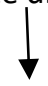

Immune response to antigenic complex

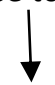

Interaction between Fas and its triggers apoptosis

pathways and cell death

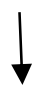

Depletion of CD4+, CD8+, T cells

ligands on epidermal cells

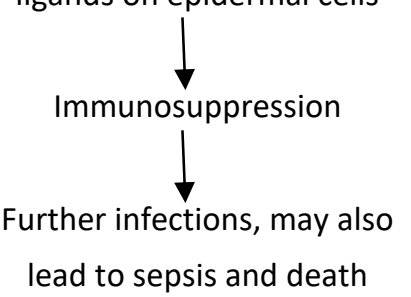

\section{CASE STUDY}

A 50 year old female patient was admitted to the Dermatology department of Tiruvallur Government hospital with chief complaints of intermittent fever with chills and rigors, rashes all over the body, loss of appetite, and painful lesions in oral cavity for the past 10days. On revealing of her medications and medical history she stated that she had had been suffering from urinary tract infection1month before. Her antibiotic medications for UTI included T. Ceftriaxone 1g, T. Nitrofurantoin 50mg, T.Trimethoprim/Sulphamethoxazole $160 / 800 \mathrm{mg}$ for the past 14 days. Her symptoms of UTI like pain with urination, pelvic pain were reduced with medications, but there was emergence of oral lesions, erythema on her hands and legs, painful ulceration lesions in the oral cavity, rashes all over the body with painful lesions. She had severe polyarthritis and arthralgia. She had a history of redness of eye with discharges while on her past medications which did not persist now. She was not a known case of Diabetes/ Hypertension/ Epilepsy/ Tuberculosis/ Coronary artery disease. On general examination the patient was conscious, oriented, afebrile with hyperpigmentation all over the body. Examination of her vitals revealed a temperature of $102^{\circ} \mathrm{F}$, pulse rate of $103 / \mathrm{min}$, respiratory rate of 20/ $\mathrm{min}$, bilateral air entry, S1 and S2 positive, soft abdomen. SGOT (90U/L) and SGPT (110U/L) levels were elevated. On assessment with Naranjo's casuality algorithm scale, the condition of SJS due to the drug was found to be probable. With regards to the clinical examination and past medication history the patient was diagnosed with Ceftriazone induced Steven Johnson's syndrome. She was immediately insisted to stop taking Ceftriazone. Her pharmacotherapy was carried out with $\mathrm{T}$. Chloroquine 250mg, Inj. Dexmethasone 50mg, Fusidic acid 250mg Prednisolone 5mg OD, Zinc cream 4mg, liquid paraffin, Zincovit tablets and other nutritional supplements.
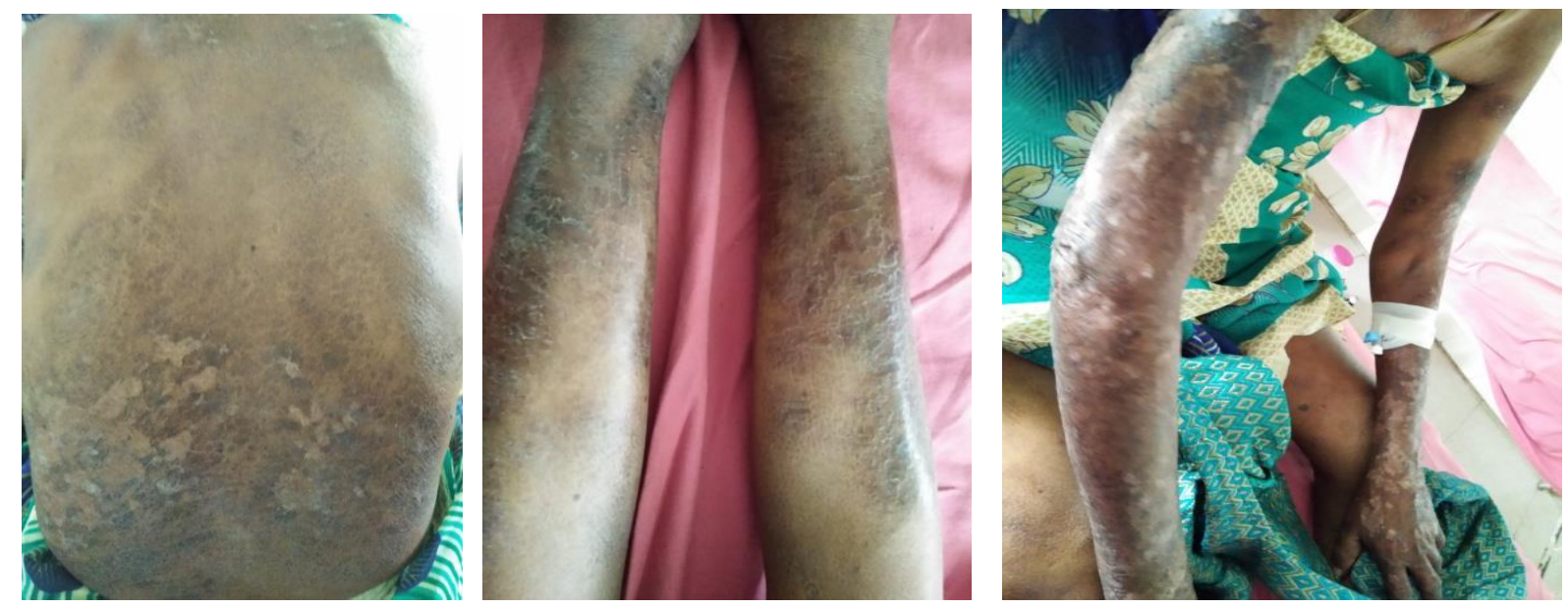

Figure 1: Lesions of Steven Johnsons syndrome observed at the trunk and Limbs of the patient
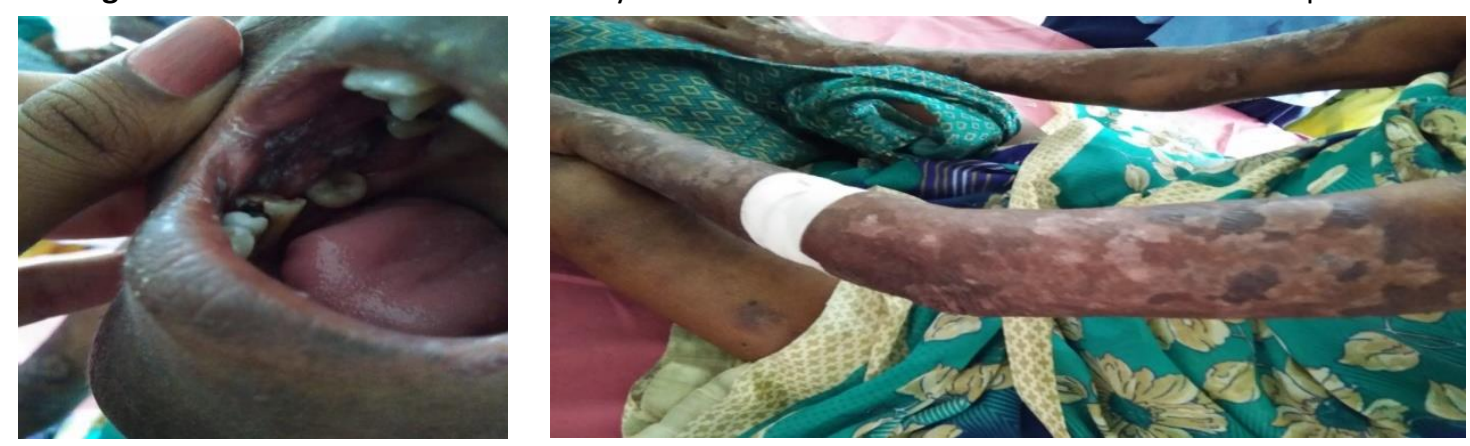

Figure 2: Oral ulcerations observed in the patient, a prominent factor 


\section{DISCUSSION}

The physical examination is assessed through the clinical presentations, signs and symptoms present within the patient. The clinical presentation would show the following;

$>$ During the systemic infection, the sepsis thereby serves as a main cause of death.

$>$ Ophthalmic complications such as conjunctivitis, purulent discharge, swollen eyelids, suppurative keratitis, pain and photophobia may also be present.

> Physiologically, pulmonary, gastro-intestinal (GI) vulvovaginitis and urologic abnormalities can also arise.

$>$ Distal portions are relatively affected but palms and soles are early site for primary lesions, which can further develop through itching, rubbing and may be due to a tender touch. Minute strawberry (or) red coloured lesions appear near thoracic areas and neck.

Pharmacist is often the first and the last provider; they have to be more active in treatment follow-up and treatment monitoring. If any primary symptoms appear they have to be reported to a health care professional or a physician about the drug and treated with anticoagulants, corticosteroids etc., often, monitoring provides a basic initial care for the patient and preventing them from complications of the associated disease $\mathrm{e}^{11,13}$.

Not only monitoring, a physician has to assess the patient with his/her past medical conditions, if any immunity abnormalities, a low and starting primary dose must be prescribed. Withdrawing the currently in-taking drug, could pave a best way for better outcomes. The prognosis such as oral ulcers and manifestations must not be ignored and has to be kept in mind while prescribing for the next case ${ }^{2,15}$. Mainly antibiotics must be prescribed with high knowledge and care, because certain antibiotics for certain type of people can produce an autoimmune disease and make them sensitive to infectious diseases. The wound dressing must be provided for the prevention against infections and corticosteroid ointment must be applied on affected areas.

Our present study is aimed to provide a case on SJS. SJS and TEN can be easily diagnosed with prior to surface area infection. Further, with drugs consumed shortly or for a longer period of time. However the drug induced SJS is more common and antibiotics accounts for major number of cases. Early diagnosis with the prompt recognition and withdrawal of causative drugs prompts favorable outcomes.

However, the syndrome is so severe and spreading; there is a lackage of potentially curative measures, this may further lead to increased mortality rate. Health care professional should monitor the drug regimen thereby if early signs are present, they should inquire for the intake of any noxious drugs. The non-essential drug or the drug inducing SJS should be withdrawn due to those bad therapeutic outcomes with ADR associated. The patients are to be immediately transferred to an ICU or burn unit. An ophthalmology consultation may also be required. In case of sepsis, there is a high possibility and risk ratio for mortality. SJS is rare but its recurrence may be future vulnerable ${ }^{1,9}$. The patients and the family are advised (or) educated to avoid consuming medications and other analogues. Other specific conditions which need to be monitored are,

- Administration of fluids and nutritional supplements intravenously in order to avoid dehydration and sepsis. Oral ulcerations are very common symptoms which make it difficult in taking of food orally.

- Correct diagnosis of the condition is essential as oral ulcers would always be misunderstood by physicians for some other conditions and patients too would avoid considering it for SJS.

- Plasmapheresis would help in draining of the culprit drug from the body, thus bringing much recovering states.

- Treatment of patient in an Intensive burn care unit would bring much beneficial conditions to the patient, as the condition almost resembles a burn state.

\section{CONCLUSION}

Generally, patients started with any common drug regimen may have a potential risk for developing SJS. Initial presenting complaint is likely to include oral erythema, ulcerations, lesions in palms and soles which a patient may ignore. Early diagnosis helps the clinician to exclude secondary infection and subsequent complications. The causative drug should be discontinued and never be rechallenged. The hypersensitivity against a specific drug directly depends upon the immunity and the immunization of the patient, for example; certain patients may develop SJS while some do not, the epidemiology has a statistical data of 2 infected in million.

\section{Acknowledgment}

The authors are thankful to Dr.C.Sekar, Chief Medical Superintendent, Government hospital, Thiruvallur for his constant support and encouragement throughout the study. We thank the patients who gave their willing consent for the publication of their case by sharing complete information needed for the study.

\section{Compliance with ethical standards}

Written informed consent was obtained from the patient for publication of the case study, inclusion of the accompanying images. Copies of written consent may be requested for review from the corresponding author.

\section{Conflict of interest}

The authors declare no conflicts of interest concerning the content of this case report. 


\section{REFERENCES}

1. Brett SA, Philips D, Lynn AW, "Intravenous immunoglobulin therapy for Stevens-Johnson Syndrome".Southern medical Journal, 94 (3), 2001, 342-3.

2. AM Stevens, FC Johnson, "A new eruptive fever associated with stomatitis and ophthalmia". Amer J Dis Child, 24(6), 1922, 526-33.

3. Dinerman $M$, "Stevens - Johnson Syndrome with Mycoplasma pneumoniae and Enterovirus", International Pediatrics. 19 (4), 2004, 237-9.

4. Bastuji-Grain S, Rzany B, Stern RS, et al, "Clinical classification of cases of toxic epidermal necrolysis, Stevens-Johnson syndrome, and erythema multiforme", Arch Dermatol. 129 (9), 1993, 1171-3.

5. Wong KC, Kennedy PJ, Lee S, "Clinical manifestations and outcomes in 17 cases of Stevens-Johnson syndrome and toxic epidermal necrolysis", Australas J Dermatol. 40(3), 1999, 1314.

6. Lebargy F, Wolkenstein P, Gisselbrecht M, et al. "Pulmonary complications in toxic epidermal necrolysis: A prospective clinical study". Intensive Care Med 23(12), 1997, 1237-44.

7. Meneux E, Paniel BJ, Pouget F, et al. "Vulvovaginal sequelae in toxic epidermal necrolysis", J Reprod Med. 42(3), 1997, 153-6.

8. Lehman SS, "Long-term ocular complication of StevensJohnson syndrome", ClinPediatr (Phila) 38 (7), 1999, 452-7.

9. Morelli MS, O'Brein FX, "Stevens-Johnson syndrome and cholestatic hepatitis", Dig Dis Sci 46(11), 2001, 2385-8.
10. Kelly JP, Auquier A, Rzany B, et al, "An international collaborative case-control study of severe cutaneous adverse reactions (SCAR), Design and methods".J ClinEpidemiol 48, 1995, 1099-08.

11. Bastuji-Garin S, Rzany B, Stern R.S, et al, "Clinical classification of cases of toxic epidermal necrolysis, Stevens-Johnson syndrome, and erythema multiforme". Arch Dermatol. 129, 1993, 92-96

12. Mockenhaupt M. "The current understanding of StevensJohnson syndrome and toxic epidermal necrolysis". Expert Review of Clinical Immunology. 7(6), 2013, 803-15.

13. Yap FBB, Wahiduzzaman M, Pubalan M. "Stevens-Johnson syndrome (SJS) and Toxic Epidermal Necrolysis (TEN) in Sarawak: A Four Years' Review". Egyptian Dermatology Online Journal. 4 (1), 2008, 1-13.

14. Roujeau JC. "Steven-Johnson syndrome and toxic epiderma necrolysis are severity variants of the same disease which differs from erythema multiforme". J Dermatol 24, 1997, 7269.

15. Letko E, Palpaliodis DN, papaliodis GN, Daound YJ, Ahmed AR, Foster CS. "Steven-Johnson syndrome and toxic epidermal necrolysis, A review of literature". Ann Allergy Asthma Immunol, 94, 2005, 419-36.

16. Roujeau JC, Kelly JP, Naldi L, Rzany B, Stern RS, Anderson T, et al. "Medication use and the risk of Stevens-Johnson syndrome or toxic epidermal necrolysis". N Engl J Med $333(24), 1995,14,1600-7$.

Source of Support: None declared.

Conflict of Interest: None declared.

For any question relates to this article, please reach us at: editor@globalresearchonline.net New manuscripts for publication can be submitted at: submit@globalresearchonline.net and submit_ijpsrr@rediffmail.com 